\title{
Proposed System for Education Augmented Reality Self English Learning
}

Renas Rajab Asaad ${ }^{1}$, Zhvan Abdulsalam Sulaiman², Suha Salim Abdulmajeed ${ }^{3}$

1,2,3College of Computer Science \& Information Technology, Nawroz University, Duhok, Kurdistan Region - Iraq

\begin{abstract}
The development of hardware technology, especially in mobile devices has provided a possibility to run large programs efficiently on smartphones such as virtual reality, augmented reality and neural network based programs. Augmented reality is a technology that works on computer vision based recognition algorithms to augment sound, video, graphics and other sensor based inputs on real world objects using the camera of your device. It is a good way to render real world information and present it in an interactive way so that virtual elements become part of the real world. In this paper there is a 3D character which will be shown in real world through camera and will teach user name of object and show that object in front of him, there is different kind of section like alphabets, fruit, animals, human organs, tools and conversation section. for example if user choose fruit the character will introduce the section at start then will show 3D model of different kind of fruits and pronounce name of each of it and so on but if user choose conversation section character will do a conversation with user like for example visiting a restaurant, the character will deal with user and behave as a waiter and start conversation and ask him like what you like to eat or drink and so on which will help user learn and figure how to deal and speak at that time. In this paper, the creation of models and character involves design, modeling and animation of the model. This is done by relying on several software and frameworks such as Autodesk Maya, Unity3D, AR foundations, etc....
\end{abstract}

Keywords: Augmented Reality, AR foundations, Self-Learning, Unity3D, Autodesk Maya, Animated Objects.

\section{Introduction}

There have been major advancements in our world in the last decade, the development of information technology has greatly affected the present. Educational institutes have been used this advanced technology for the purpose of educations for student. In early days, education was with books, blackboard, choke, etc.. which was enough at that time to prepare students for real world. But in nowadays, the young generation are living in a very different reality as it almost all based on technology, so it is very beneficial to use advanced technologies such as augmented reality (AR) to help students focus and understand during the courses, this further enhanced their comprehension and visualization of subjects. AR and VR have been previously used in

Academic Journal of Nawroz University

(AJNU) Volume 8, No 3 (2019).

Regular research paper : Published 31 Aug 2019

Corresponding author's e-mail : renas_rekany@yahoo.com

Copyright $@ 2017$ Renas Rajab Asaad1', Zhvan Abdulsalam

Sulaiman ${ }^{2}$, Suha Salim Abdulmajeed ${ }^{3}$. This is an open access article distributed under the Creative Commons Attribution License. education but it wasn't used for public at start as it was used in U.S. Air Force Research and in NASA x-38 aircraft. In 1996 Christine M. Byrne developed a virtual reality software called Water on Tap during his $\mathrm{PhD}$ course which was about an immersive virtual reality environment for chemistry education ${ }^{[1]}$. AR is a technology that blends three dimensional objects with the user's environment in real time. Augmented reality is different from virtual reality in that VR establishes a computer created environment for the user to experience. While AR is the real world user environment extended with computerized objects. Also there is Mixed Reality which is combination of real world with virtual world. The two worlds are "mixed" together to create a realistic environment. A user can navigate this environment and interact with both real and virtual objects ${ }^{[2]}$. This paper presents an idea of self-learning method to learn English language without needing any special tutor. In this paper there is many different kind of sections in it, each section has its own objects that related only to that section like fruit which has many different $3 \mathrm{~d}$ models of fruits that will be shown in front of user and beside of objects there is a character which appears when user get into the 
section. The character introduce section and talks about section content and what user should learn from this section and it pronounce name of each models and translate it for user, when character talks about a specific object the model of that object get animated and move from its start location toward user and get scaled and rotating until character finishing talking about it then it return back to its origin location and next model will go through same process and so on until all models finish. The proposed application is built using several technologies. First the character design and animation are created with iClone Character Creator. iClone Character Creator is centered to complete the 3D game character pipeline for the industry. Other than adding a highly optimized game character base for mobile, AR and VR developers ${ }^{[3]}$. Second design of each section objects except character are created with Maya3D. Maya3D is a modeling and animation software that is used to create a variety of objects, animate them and render them ${ }^{[4]}$. Third Unity3D has been used to create the scene of each section, timeline for animations and make use of AR foundations augmented reality framework. Unity3D is a gaming engine, it can create powerful and efficient $\mathrm{AR}$ and VR experiences ${ }^{[5]}$. AR foundations is a combination of ARCore and ARKit platforms which are for developing augmented reality application for iOS and Android. It enables detection of vertical and horizontal planes, as well as $3 \mathrm{~d}$ and $2 \mathrm{~d}$ marker ${ }^{[6]}$.

\section{Literature Review}

With this project we used a main technology which is augmented reality to present $3 \mathrm{D}$ objects in real world using smartphones camera only for this purpose we used AR Foundation as it is cross platform API that works on different platform like iOS and Android with same code and same project.

\subsection{Augmented Reality (AR)}

Augmented reality is the technology that expands our physical world, adding layers of digital information onto it. Unlike Virtual Reality (VR), AR does not create the whole artificial environments to replace real with a virtual one. AR appears in direct view of an existing environment and adds sounds, videos, graphics to it.

A view of the physical real-world environment with superimposed computer-generated images, thus changing the perception of reality, is the AR.

The term itself was coined back in 1990, and one of the first commercial uses were in television and military. With the rise of the Internet and smartphones, AR rolled out its second wave and nowadays is mostly related to the interactive concept. 3D models are directly projected onto physical things or fused together in real-time, various augmented reality apps impact our habits, social life, and the entertainment industry.[7]

AR apps typically connect digital animation to a special 'marker', or with the help of GPS in phones pinpoint the location. Augmentation is happening in real time and within the context of the environment, for example, overlaying scores to a live feed sport event.

There are 4 types of augmented reality today:

- Marker less AR

- Marker-based AR

- Projection-based AR

- Superimposition-based AR

\subsection{AR Foundation}

Unity, maker of the eponymous game engine, continues to advance its AR Foundation project, which aims to make it easier for developers to create AR apps that runs both on iOS and Android. Its latest release adds support for ARKit's ARWorldMap and Unity's Lightweight Render Pipeline. ${ }^{[8]}$

AR Foundation exposes a common API which aims to cover the core functionality of both Android ARCore and iOS ARKit, thus making it possible to create AR apps for both platforms from a single code base. After providing support for a number of basic AR features in its first release, including plane detection, device position and orientation tracking, light estimation, and others, Unity is now adding more advanced features to its offerings.

One of those is support for ARKit ARWorldMap, which enables the creation of shared or persistent experience. Shared experience allows multiple users to see and interact with the same AR scene using different devices at the same time, with each user seeing the common virtual environment from their own perspective. ARWorldMap also makes it possible to create persistent AR experience that can be stored and recreated at some other point in time. Another ARKit feature that is now supported by AR Foundation is face tracking, which makes it possible to track the movement and expressions of the user's face. It is worth noting that both world map and face tracking support are for the time being exclusive to ARKit.

\subsection{ARCore}

Is Google's platform for building augmented reality experiences. Using different APIs, ARCore enables your phone to sense its environment, understand the world and interact with information. Some of the APIs are available across Android and iOS to enable shared AR experiences. ${ }^{[9]}$ 
ARCore uses three key capabilities to integrate virtual content with the real world as seen through your phone's camera:

- Motion tracking allows the phone to understand and track its position relative to the world.

- Environmental understanding allows the phone to detect the size and location of all type of surfaces: horizontal, vertical and angled surfaces like the ground, a coffee table or walls.

- Light estimation allows the phone to estimate the environment's current lighting conditions.

\subsection{ARkit}

is the framework from Apple that handles the processing to built Augmented Reality apps and games for iOS devices. It is a high-level API supplying numerous and powerful features making a magical world come to life.[10]

ARKit can be divided into 3 layers. Those layers works together simultaneously.

\subsubsection{Tracking}

is the key function of ARKit. It allows us to track a device's position, location and orientation in the real world and live.

\subsubsection{Scene Understanding}

Understanding the scene means that ARKit analyzes the environment presented by the camera's view, then adjust the scene or provide information on it. This is what enables the detection of all the surfaces in the physical world such as the floor or a flat surface. Then, it will allow us to place a virtual object on it. Also, the light estimation can be integrated to lit a virtual object simulating a light source in the physical world.

\subsubsection{Rendering}

ARKit uses technologies to handle the processing of the 3D models and present them in your scene such as:

- Metal

- SceneKit

- Third party tools like Unity or Unreal Engine

\subsection{Augmented Reality Devices}

Many modern devices already support Augmented reality. From smartphones and tablets to gadgets like Google Glass or handheld devices, and these technologies continue to evolve. For processing and projection, AR devices and hardware, first of all, have requirements such as sensors, cameras, accelerometer, gyroscope, digital compass, GPS, CPU, displays, and things we've already mentioned.[11]

\section{Implementation}

The application design and created by using different software its object designed in Maya and sounds are recorded and edited by using Adobe Audition and character designed and animated in iClone also objects are animated in Unity then coded using C\# to detect surface and putting object in real world.

\subsection{Modeling Objects}

Each scene in this application has its own objects which are modeled in Maya and exported to Unity as shown in the following figures:

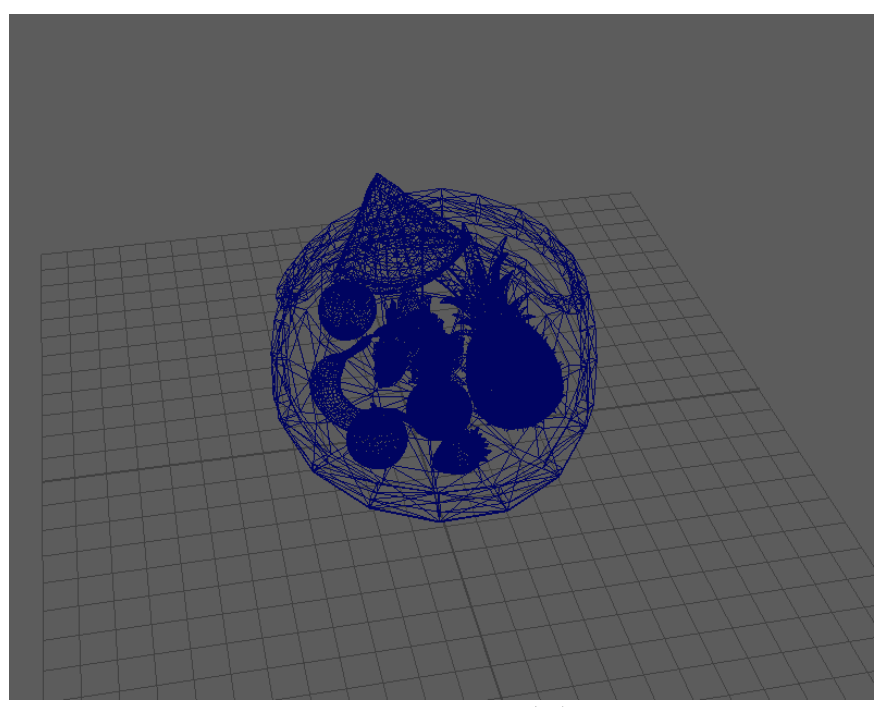

Fig 1: Fruit Model 1

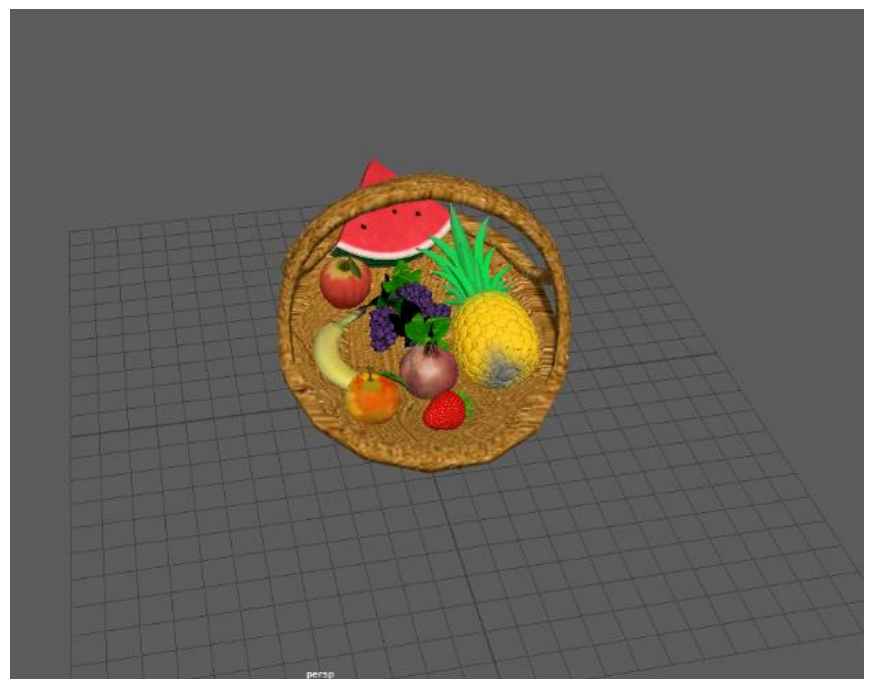

Fig 2: Fruit Model 2 


\subsection{Animating Objects}

Animating objects using unity animation tools as unity is very powerful animation tools as shown in the following figure:

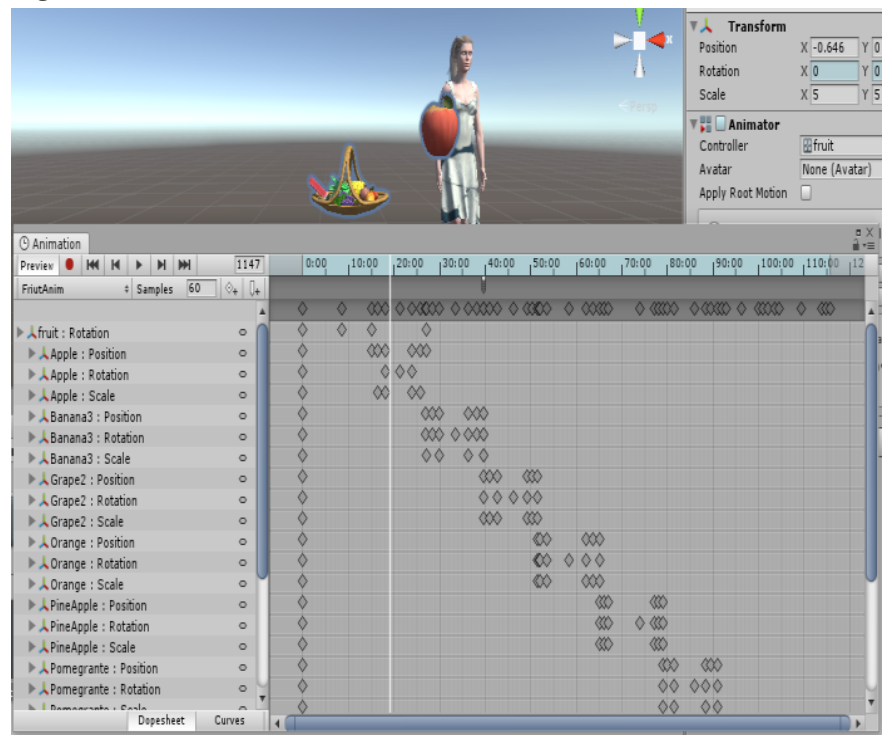

Fig 3: Animation Timeline

\subsection{Recording and Editing Sounds}

Recording sound of each object in the scene using Adobe Audition and then editing and adding effect to perfectly proper the scene the following figures show sounds wave from Adobe Audition software.

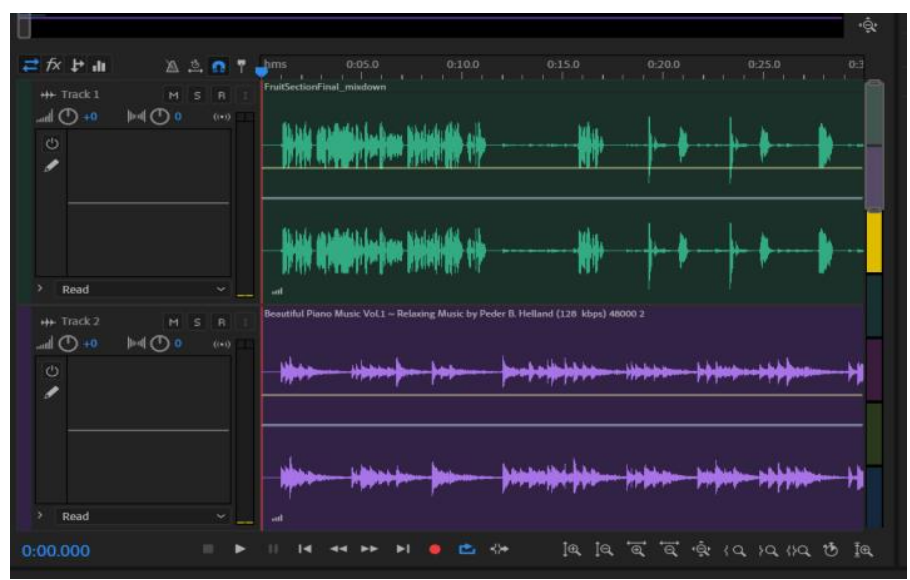

Fig 4: Multitrack Session

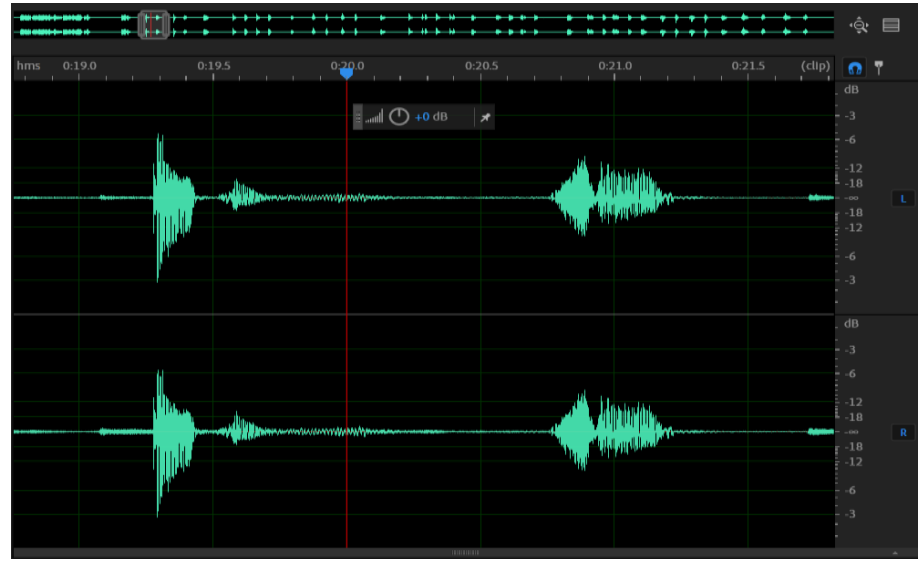

Fig 5: Single Track Waves

\subsection{Character Animations}

Creating character and rigging using Maya and adding animations by using iClone software ${ }^{[12]}$ as shown in below figure:

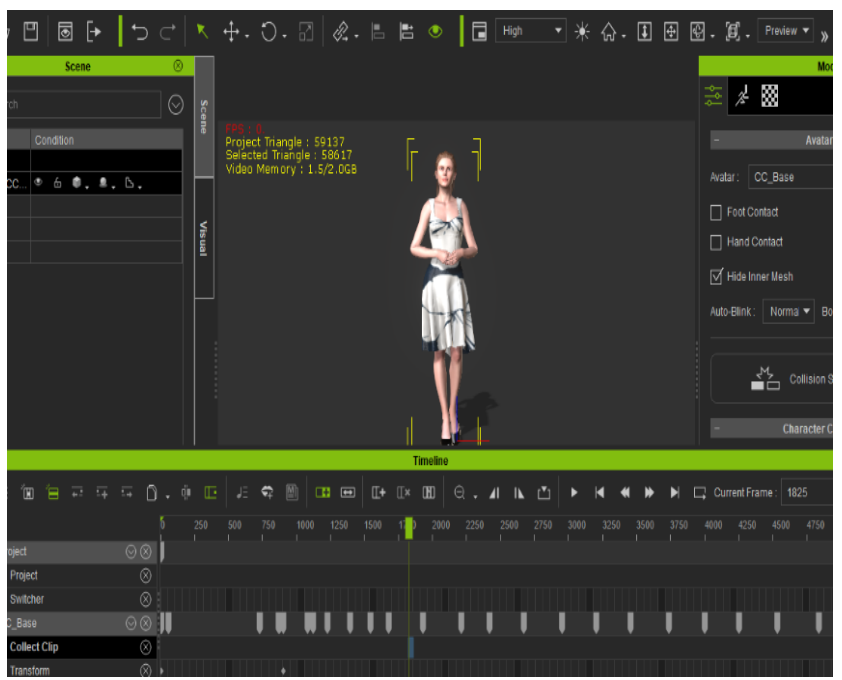

Fig 6: iClone Animating Character

\subsection{Application Main Menu User Interface}

Creating main menu to go through different section of project with a friendly user interface using Unity UI component as it shown in the following figure:

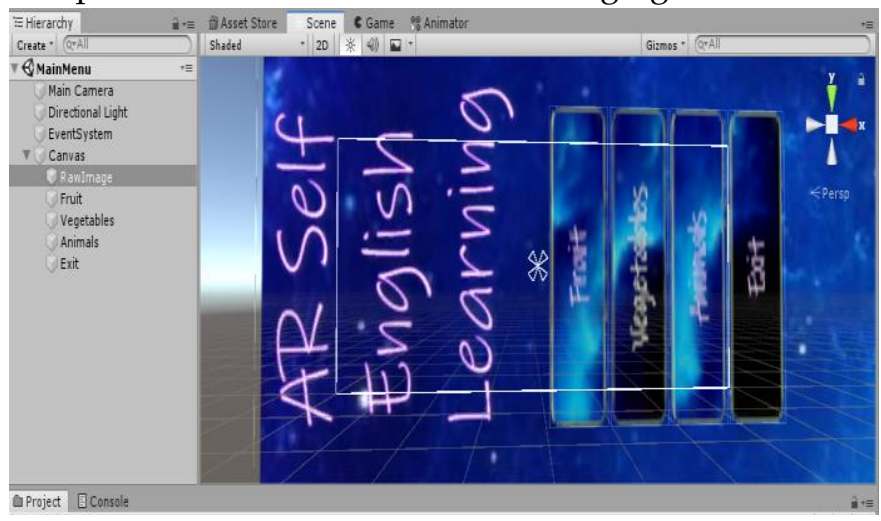

Fig 7: Main Menu User Interface 


\subsection{Placing Objects Script}

Tap to place C\# Script to place object on detected surface ${ }^{[13]}$ as showing in following code:

public class ARTapToPlaceObject : MonoBehaviour

\{ public GameObject objectToPlace;

private ARSessionOrigin arOrigin;

private Pose placementPose;

private bool placementPoseIsValid = false;

private bool objectAppeard $=$ false;

void Start()

$\{$ arOrigin $=$

FindObjectOfType<ARSessionOrigin $>()$;

\}

void Update()

$\{\quad$ UpdatePlacementPose();

if (placementPoseIsValid \&\& Input.touchCount $>0$ $\& \&$ Input.GetTouch(0).phase $==$ TouchPhase $\cdot$ Began $\& \&$ !objectAppeard)

\section{$\{\quad$ PlaceObject();}

objectAppeard = true;

\}

\}

private void PlaceObject()

$\{$ Instantiate(objectToPlace,

placementPose.position, placementPose.rotation);

\}

private void UpdatePlacementPose()

$\{$ var screenCenter $=$

Camera.current.ViewportToScreenPoint(new

Vector3 $(0.5 f, 0.5 f))$;

var hits $=$ new List $<$ ARRaycastHit $>()$;

arOrigin.Raycast(screenCenter, hits,

TrackableType.Planes);

placementPoseIsValid $=$ hits. Count $>0$;

if (placementPoseIsValid)

$\{\quad$ placementPose $=$ hits[0].pose; var cameraForward $=$

Camera.current.transform.forward;

var cameraBearing $=$ new

Vector3(cameraForward.x, 0 ,

cameraForward.z).normalized;

placementPose.rotation $=$

Quaternion.LookRotation(cameraBearing);

\}$\}$

\section{Conclusion}

In conclusion, the advancement and price of hardware has enabled running such technology on devices at our disposal already. Technologies like virtual and augmented reality can have a large variety of benefits in the present time. specially in educational processs, where it is hard to acquire the materials, tools and equipment required for object for best experimentation.

In this paper a character act like a for user which will save him an effort, time and tuition cost as he can access to his class in any time and any place, also there is conversation section which is most important as it help user to have courage and broke his shame ice wall and try to speak because he feels more comfortable as he know he is dealing with only a virtual character than dealing with real person. There is different kind of section when user study a specific section it helps him to save new words easily as it shows him the 3D model of object and pronounce it names at same time which helps him to memorize better than only repeating a word orally.

\section{REFERENCES}

[1] O. Pasaréti, H. Hajdú, T. Matuszka, A. Jámbori, I. Molnár and M. Turcsányi-Szabó, "Augmented Reality in education," in Informatika Szakmódszertani Konferencia, 2011.

[2] K. Lee, "Augmented Reality in Education and Training," TechTrends, vol. 56, no. 2, pp. 13-21, 2012..

[3] Reallusion Inc., "iClone," 2019. [Online]. Available: https://www.reallusion.com/charactercreator/production.html

[4] Autodesk Inc., "Maya3D," 2018. [Online]. Available: https://www.autodesk.com/products/maya/o verview.

[5] Unity3D, "Unity3d," 2018. [Online]. Available: 
https://unity3d.com/unity.

[6] Unity3D, "AR Foundation," 2018. [Online].

Available:

https://docs.unity3d.com/Packages/com.unity

.xr.arfoundation@1.0/manual/index.html

[7] Latta, S., McCulloch, D., Scott, J., \& Geisner, K. (2014). U.S. Patent Application No. 13/670,258.

[8] Glover, Jesse. Unity 2018 Augmented Reality

Projects: Build four immersive and fun AR applications using ARKit, ARCore, and Vuforia. Packet Publishing Ltd, 2018.

[9] Linowes, J., \& Babilinski, K. (2017). Augmented

Reality for Developers: Build practical

augmented reality applications with Unity, ARCore, ARKit, and Vuforia. Packt Publishing

Ltd.

[10] Wang, Wallace. Beginning ARKit for IPhone and IPad: Augmented Reality App

Development for IOS. Apress, 2018.

[11] Lee, Bongshin, et al. "Watches to Augmented Reality: Devices and Gadgets for Data-Driven Storytelling." Data-Driven Storytelling. AK Peters/CRC Press, 2018. 135-149.

[12] Palamar, Todd. Mastering Autodesk Maya 2016: Autodesk Official Press. John Wiley \& Sons, 2015.

[13] Savu, Laura. "DEVELOPING VIRTUAL REALITY AND AUGMENTED REALITY PROJECTS WITH UNITY3D." Journal of Information Systems \& Operations Management 12.2 (2018): 431-447. 